\title{
Cigarette Smoke Reduces Fatty Acid Catabolism, Leading to Apoptosis in Lung Endothelial Cells: Implication for Pathogenesis of COPD
}

\author{
Jiannan Gong, Hui Zhao, Tanzhen Liu, Lifang Li, Erjing Cheng, Shuyin Zhi, Lufei Kong, \\ Hong-Wei Yao* and Jianqiang Li *
}

Department of Respiratory and Critical Care Medicine, The Second Hospital of Shanxi Medical University, Taiyuan, China

\section{OPEN ACCESS}

Edited by:

Paolo Montuschi,

Catholic University of the

Sacred Heart, Italy

Reviewed by:

Oren Rom,

University of Michigan,

United States

Dolors Serra,

University of Barcelona,

Spain

Weiliang Qiu,

Sanofi Genzyme,

United States

*Correspondence:

Jianqiang $\mathrm{Li}$

ljahx@sina.com

Hong-Wei Yao

mikeyao0215@hotmail.com

Specialty section:

This article was submitted to Respiratory Pharmacology, a section of the journal

Frontiers in Pharmacology

Received: 30 April 2019

Accepted: 24 July 2019

Published: 29 August 2019

Citation:

Gong J, Zhao H, Liu T, Li L, Cheng E,

Zhi S, Kong L, Yao H-W and LiJ

(2019) Cigarette Smoke Reduces

Fatty Acid Catabolism, Leading to

Apoptosis in Lung Endothelial Cells:

Implication for

Pathogenesis of COPD.

Front. Pharmacol. 10:941.

doi: 10.3389/fphar.2019.00941
Endothelial cell (EC) apoptosis contributes to cigarette smoke (CS)-induced pulmonary emphysema. Metabolism of glucose, glutamine, and fatty acid is dysregulated in patients with chronic obstructive pulmonary disease (COPD). Whether CS causes metabolic dysregulation in ECs leading to development of COPD remains elusive. We hypothesized that CS alters metabolism, resulting in apoptosis in lung ECs. To test this hypothesis, we treated primary mouse pulmonary microvascular ECs (PMVECs) with CS extract (CSE) and employed PMVECs from healthy subjects and COPD patients. We found that mitochondrial respiration was reduced in CSE-treated PMVECs and in PMVECs from COPD patients. Specifically, oxidation of fatty acids (FAO) was reduced in these cells, which linked to reduced carnitine palmitoyltransferase 1a (Cpt1a), an essential enzyme for carnitine shuttle. CSE-induced apoptosis was further increased when cells were treated with a specific Cpt1 inhibitor etomoxir or transfected with Cpt1a siRNA. L-Carnitine treatment augmented FAO but attenuated CSE-induced apoptosis by upregulating Cpt1a. CSE treatment increased palmitate-derived ceramide synthesis, which was reduced by L-carnitine. Although CSE treatment increased glycolysis, inhibiting glycolysis with 2-deoxy-D-glucose had no effects on CSE-mediated apoptosis in lung ECs. Conclusively, FAO reduction increases ceramide and apoptosis in lung ECs treated with CSE, which may contribute to the pathogenesis of COPD/emphysema.

Keywords: chronic obstructive pulmonary disease, endothelial cells, metabolism, ceramide, apoptosis

\section{INTRODUCTION}

Chronic obstructive pulmonary disease (COPD) is a progressive, obstructive disease of the lungs, and it becomes the third leading cause of death worldwide (Rennard and Drummond, 2015). The pathology of this disease is characterized by abnormal inflammatory responses, mucus hypersecretion, airway obstruction and remodeling, as well as alveolar destruction (Mirza et al., 2018). Cigarette smoke (CS) is a major risk factor for the development of COPD, which accounts for

Abbreviations: COPD, chronic obstructive pulmonary disease; Cpt, carnitine palmitoyltransferase; CS, cigarette smoke; CSE, cigarette smoke extract; ECAR, extracellular acidification rate; ECs, endothelial cells; FAO, fatty acid oxidation; PMVECs, lung microvascular endothelial cells; OCR, oxygen consumption rate. 
at least $75 \%$ of COPD deaths. CS contains thousands of chemicals, which contact with lung epithelial cells and damage them directly. This leads to cascade responses, including oxidative stress, protease/antiprotease imbalance, inflammation, apoptosis, and senescence, thereby causing lung injury (Yao and Rahman, 2011; Yue and Yao, 2016). In addition to the epithelial cells, endothelial cells (ECs) are also effector cells during CS-induced emphysema, as the volatile components in CS, such as aldehyde and acrolein, can directly enter into the blood vessels, causing EC injury (Kim et al., 2016; Voelkel, 2018). In fact, EC apoptosis is one of the contributing factors to the development of pulmonary emphysema (Petrache et al., 2005; Giordano et al., 2008; Garcia-Lucio et al., 2018). However, the mechanisms underlying CS-induced EC apoptosis are not fully understood.

Previous studies have shown that glucose, fatty acids, and glutamine metabolism are abnormal in patients with COPD (Kao et al., 2012; Wang et al., 2013; Telenga et al., 2014; Cruickshank-Quinn et al., 2018). These studies employed metabolomics, lipidomics, and metabolic flux to determine fuel metabolism using sputum, blood, and urine samples from patients with COPD.

These approaches provide advanced technology to detect a complete picture of the metabolic phenotype from COPD patients. For example, glucose production, clearance, and oxidation, and rate of glycolysis were increased in patients with COPD compared with control subjects. However, there is no change in the rate of nonoxidative disposal of pyruvate or serum lactate between healthy controls and COPD patients. (Kao et al., 2012). Lipid metabolism was dysregulated during the onset of COPD (Jiang et al., 2017). Glycerophospholipid and sphingolipid metabolism were associated with worse airflow obstruction, lung function decline, and COPD exacerbations (Cruickshank-Quinn et al., 2018). Compared to healthy controls, COPD patients displayed decreased lipoprotein and amino acids in serum and urine (Wang et al., 2013), including branched-chain amino acids, and increased glycerolphosphocholine in serum. Nevertheless, the cell-specific metabolic alterations in COPD remain elusive. CS exposure has been shown to reduce glycolysis in type II cells (Agarwal et al., 2014). Furthermore, acute CS exposure was shown to induce a switch from glucose to lipid as the main energy source and increase FAO in distal lung epithelial cells. It is unclear whether CS modulates metabolism in lung ECs. In this study, we hypothesize that CS alters metabolism in lung ECs, thereby causing apoptosis. Mitochondrion is a key organelle, which links to metabolism with apoptosis. Therefore, we tested this hypothesis to determine mitochondrial respiration and fuel utilization as well as its association with apoptosis in lung ECs treated with CS extract (CSE).

\section{MATERIALS AND METHODS}

\section{Cell Culture}

Human pulmonary microvascular ECs (PMVECs) from healthy subjects and COPD patients with smoking history were obtained from Lonza (Walkersville, MD) and Cell Biologics (Chicago, IL). Cells were cultured in complete microvascular EC growth medium supplemented with the SingleQuots (Lonza). Culture medium was changed every $48 \mathrm{~h}$. Cells (less than passage 6) with $70-80 \%$ confluency were used for experiments. Cells from each group were pooled for further treatment and analysis.

\section{Mouse PMVEC Isolation}

Primary PMVECs were isolated as described previously with modifications (Suresh et al., 2017; Yao et al., 2019). C57BL/6J mice ( $8-10$ weeks old, both male and female) were anesthetized, and lungs were removed. A mouse lung dissociation kit (Miltenyi Biotec) was used to enzymatically digest lungs. After removal of CD $45^{+}$cells, CD $45^{-}$cells were collected, washed, and incubated with CD31-conjugated beads (Invitrogen). CD31 ${ }^{+}$cells were enriched using a MACS column and magnetic field. The freshly isolated cells were considered as passage 0 , which cultured in dish coated human fibronectin $(30 \mu \mathrm{g} / \mathrm{ml})$. Cells that were less than passage 5 were used for experiments. All animals were housed in accordance with the Guide for the Care and Use of Laboratory Animals. The animal study was approved by the Animal Care and Ethics Committee of the Second Hospital of Shanxi Medical School (CMTT\#: 2013012) in accordance with international standards.

\section{CSE Preparation}

Smoking from a Kentucky research 3R4F cigarette was bubbled into $10 \mathrm{ml}$ of culture medium by a negative pressure pump at a speed of $1 \mathrm{~min}$ per cigarette. The optical density of this aqueous extract of smoking was measured at a wave length of $320 \mathrm{~nm}$ and adjusted into 1, which was considered as 10\% CSE based on previous reports (Caito et al., 2010; Cui et al., 2018). The CSE was filtered through a $0.2-\mathrm{mm}$ sterile filter to remove bacteria. Before each experiment, the CSE was freshly prepared so as to avoid the breakdown of aqueous substances and evaporation of volatile components. Control medium was prepared using air rather than smoking with the same procedure.

\section{Cell Treatment and Transfection}

Cells were treated with L-carnitine $(1 \mathrm{mM})$, etomoxir $(10 \mu \mathrm{M})$, myriocin $(100 \mathrm{nM})$, or palmitate-bovine serum albumin (BSA) $(50 \mu \mathrm{M})$ based on preliminary studies and publications (Yao et al., 2019). Mouse PMVECs cells were cultured onto a six-well plate with the density of $1 \times 10^{6} /$ well. Carnitine palmitoyltransferase 1a (Cptla) siRNA (Cat\#: AM16708, IDs: 161677 and 161678, Thermo Fisher Scientific) at $50 \mathrm{nM}$ was added with the Lipofectamine 2000 (Invitrogen) for $24 \mathrm{~h}$ to reduce endogenous Cptla expression. In each well, the volume of siRNA and Lipofectamine 2000 was 5 and $5 \mu$, respectively.

\section{Viability Assay}

Cell viability was assessed after the addition of 3-(4,5-dimethylthiazol-2-yl)-2,5-diphenyl tetrazolium bromide (MTT, $4 \mathrm{mg} / \mathrm{ml}$ ) for the final $5 \mathrm{~h}$. MTT incorporation was measured at a $570-\mathrm{nm}$ wavelength by a microplate absorbance reader (Biorad-550). 


\section{Measurement of Oxygen Consumption Rate and Substrate Oxidation}

Real-time oxygen consumption rate (OCR) was recorded using the Seahorse XF-24 Analyzer (Seahorse Bioscience). Cells at 15,000 cells per well were seeded in a Seahorse culture microplate. OCR was determined after sequential injections with oligomycin $(1 \mu \mathrm{M})$, carbonyl cyanide p-trifluoromethoxyphenylhydrazone (FCCP, $0.5 \mu \mathrm{M})$, and rotenone/antimycin $\mathrm{A}(0.5 \mu \mathrm{M})$ according to the manufacturer's manual. The numbers of cells in well was used to normalize the OCR values. Mitochondrial oxidation of fatty acids, glucose, and glutamine were determined by the Seahorse Analyzer following the instruction of the Seahorse XF Mito Fuel Flex Test kit (Pike Winer and $\mathrm{Wu}, 2014)$. In brief, etomoxir $(4 \mu \mathrm{M}), 2$-cyano3-(1-phenyl-1H-indol-3-yl)-2-propenoic acid (UK5099, $2 \mu \mathrm{M}$ ), and bis-2-(5-phenylacetamido-1,3,4-thiadiazol-2-yl)ethyl sulfide (BPTES, $3 \mu \mathrm{M}$ ) were sequentially injected from the cartridge plate during the OCR measurement. Etomoxir, UK5099, and BPTES are specific inhibitors of fatty acid, glucose, and glutamine oxidation, respectively. The substrate oxidation utilization was calculated as the percentage of specific pathway utilization to the whole OCR levels.

\section{Mass Spectrometry Analysis}

To directly measure fatty acid oxidation (FAO), cells were incubated with medium supplemented with $100 \mu \mathrm{M}\left[\mathrm{U}-{ }^{13} \mathrm{C}\right]$ palmitate or palmitate conjugated to BSA for $6 \mathrm{~h}$ (Yao et al., 2016). Palmitate/ BSA conjugation was performed when $1 \mathrm{mM}$ sodium palmitate (Sigma) or $1 \mathrm{mM}$ potassium-U-13C palmitate (Cambridge Isotopes) was conjugated with $0.17 \mathrm{mM}$ fatty acid free-BSA (Sigma) in $150 \mathrm{mM} \mathrm{NaCl}$ solution at $37^{\circ} \mathrm{C}$ for $1 \mathrm{~h}$. We extracted cells as previously described (Vaskovsky et al., 1975; Ivanisevic et al., 2013). The Agilent 6530 Q-TOF was used to identify and analyze levels of citrate, $\alpha$-ketoglutarate, fumarate, malate, and ceramide, which was confirmed by comparing retention times and tandem mass spectrometry data with standard compounds. The results were corrected for naturally occurring ${ }^{13} \mathrm{C}$ impurity of the tracers.

\section{RNA Extraction and Real-Time Polymerase Chain Reaction}

RNeasy miniprep kit (Qiagen) was used to purify DNA after extraction by the TRIzol reagent. RNA concentrations were measured by the NanoDrop spectrophotometer. We used $500 \mathrm{ng}$ of total RNAs for reverse transcription with the Taqman ${ }^{\circledR}$ Reverse Transcription Reagents (ThermoFisher Scientific). Real-time polymerase chain reaction (PCR) was performed using $1 \mu \mathrm{l}$ of cDNA by the 7300 Real-Time PCR System (Applied Biosystems). All TaqMan gene probes were purchased from the Thermo Fisher Scientific. Mm01231183, Mm00487191, and Mm00463970 are the catalog numbers for Cpt1a, Cpt1b, and Cpt1c probes, respectively. 18s rRNA was used as a housekeeping gene for normalization. RNA levels were calculated by the comparative $2^{-\Delta \Delta C t}$ method.

\section{Western Blot}

Cells were lyzed with $0.3 \mathrm{ml}$ of radioimmunoprecipitation assay buffer $(50 \mathrm{mmol} / \mathrm{l}$ Tris- $\mathrm{HCl}, 150 \mathrm{mmol} / \mathrm{l} \mathrm{NaCl}, 1 \mathrm{mmol} / \mathrm{l}$ EDTA, $0.25 \%$ deoxycholate, $1 \mathrm{mmol} / \mathrm{l} \mathrm{NaF}, \quad 0.5 \mathrm{mmol} / \mathrm{l}$
$\mathrm{Na}_{3} \mathrm{VO}_{4}, 1 \mathrm{mg} / \mathrm{l}$ aprotinin, $1 \mathrm{mg} / \mathrm{l}$ leupeptin, and $0.5 \mathrm{mmol} / \mathrm{l}$ phenylmethylsulfonyl fluoride). We kept the lysates on ice for $30 \mathrm{~min}$ and then passed them three times through a 22-gaugue needle to disassociate the DNA. Whole cell lysates were centrifuged at $10,000 \mathrm{rpm}$ in an Eppendorf tube for $5 \mathrm{~min}$ in $4^{\circ} \mathrm{C}$, and the supernatant was collected. A bicinchoninic acid (BSA) Protein Assay kit (Thermo Scientific, Rockford, IL, USA) was used to measure protein levels in supernatants. Samples (10-20 $\mu$ g proteins) were separated on a $10 \%$ sodium dodecyl sulfate-polyacrylamide gel electrophoresis gel (Invitrogen), and separated proteins were electroblotted onto nitrocellulose membranes. The blots were blocked for $1 \mathrm{~h}$ at room temperature with $5 \%$ milk, and we probed them with 1:1,000 diluted antibody against Cptla (Cat\#: ab128568, Cell Signaling). The abundance of protein was determined using secondary antibodies with 1:10,000 dilutions linked to horseradish peroxidase. The ChemiDoc ${ }^{\mathrm{TM}}$ Imaging System was used to detect the band with the enhanced chemiluminescence method. $\beta$-Actin was used as a housekeeping control.

\section{Flow Cytometric Assay for Annexin V-Positive Cells}

Flow cytometry was used to measure Annexin V-positive cells that are considered as apoptotic cells. Briefly, cells were collected using the TrypLE (Thermo Fisher Scientific). After washed with cold phosphate buffered saline, Annexin $\mathrm{V}$ binding buffer $(300 \mu \mathrm{l})$ was added to resuspend cells. A total $0.1 \times 10^{6}$ cells were collected and incubated with $100 \mu$ Annexin $\mathrm{V}$ binding buffer along with $5 \mu$ l of fluorescein isothiocyanate conjugated Annexin V (Life Technologies, USA) and $5 \mu \mathrm{l}$ of propidium iodide (Life Technologies, USA) for $20 \mathrm{~min}$ at room temperature. Finally, we added Annexin V binding buffer $(500 \mu \mathrm{l})$ and mixed gently. FC-500 (Beckman Coulter) was used to detect the Annexin V-positive cells with total 20,000 events analyzed.

\section{Statistical Analysis}

Experiments were performed at least three biological replicates with total $n=5-6$ times of measurements. Data are expressed as mean \pm SEM. One-way analysis of variance (ANOVA) was used to determine whether there are any statistical significance between the means of groups. The Student-Newman-Keuls (SNK) test was used to examine which specific groups of means were statistically different. Statistical significance was considered when $P<0.05$.

\section{RESULTS}

\section{Oxidative Phosphorylation Was Reduced in CSE-Treated Mouse PMVECs and in PMVECs From COPD Patients}

CS has been shown to reduce mitochondrial respiration in alveolar epithelial cells (Agarwal et al., 2014). It is unknown whether CS alters mitochondrial respiration in lung ECs. To answer this question, we determined the effect of CSE on mitochondrial respiration in primary mouse PMVECs. We first treated cells with CSE $(0.1,0.25$, and $0.5 \%)$ for 6,12 , and $24 \mathrm{~h}$ and found that CSE reduced cell viability in a dose- and time-dependent 
manner (Figure 1A). Thus, we chose CSE $(0.25 \%, 12 \mathrm{~h})$, which may impair cell function (e.g., apoptosis) but not affect cell viability, for following experiments. Treatment with CSE $(0.25 \%$, $12 \mathrm{~h}$ ) significantly reduced oxidative phosphorylation in mouse PMVECs, which was shown by the decreased basal and maximal respiration compared to control group (Figure 1B). Similarly, the OCR was decreased in PMVECs from COPD patients (Figure 1C). Once normalized to their corresponding basal respiration, we did not observe any significant changes in kinetic response of mitochondrial respiration between air and CSE groups (Figure 1D). This suggests that CS exposure results in a reduction in mitochondrial respiration, particularly in basal respiration.

\section{FAO Was Reduced in CSE-Treated Mouse PMVECs and in PMVECs From COPD Patients}

To determine whether CS exposure alters substrate utilization by mitochondria, we determined the oxidation of glucose, glutamine, and long-chain FA in human PMVECs using the Mitochondrial Fuel Flex Test kit. We found that the percentage of FAO was reduced in human PMVECs treated with CSE $(0.25 \%)$ for $12 \mathrm{~h}$. There were no changes in glucose or glutamine oxidation in human PMVECs treated with CSE (0.25\%) for $12 \mathrm{~h}$ (Figure 2A). Furthermore, the utilization of FAs was significantly reduced in PMVECs from COPD patient as compared to healthy subject (Figure 2B). To further directly determine the oxidation of FAs, we treated human PMVECs with $\mathrm{U}_{-13}{ }^{13} \mathrm{C}$-labeled palmitate and measured the levels of citrate, a-ketoglutarate, fumarate, and malate derived from exogenous palmitate. As shown in Figure 2C, the levels of palmitate-derived citrate, a-ketoglutarate, fumarate, and malate were significantly reduced in human PMVECs treated with CSE $(0.25 \%, 12 \mathrm{~h})$ compared to air control. Altogether, these results demonstrate that FAO is reduced in human PMVECs exposed to CS.

\section{Cpt1a Levels Was Reduced in CSE-Treated Mouse PMVECs and in PMVECs From COPD Patients}

Carnitine shuttle transfers the acyl group of long-chain FAs into mitochondria, where Cpt1 is an essential enzyme for

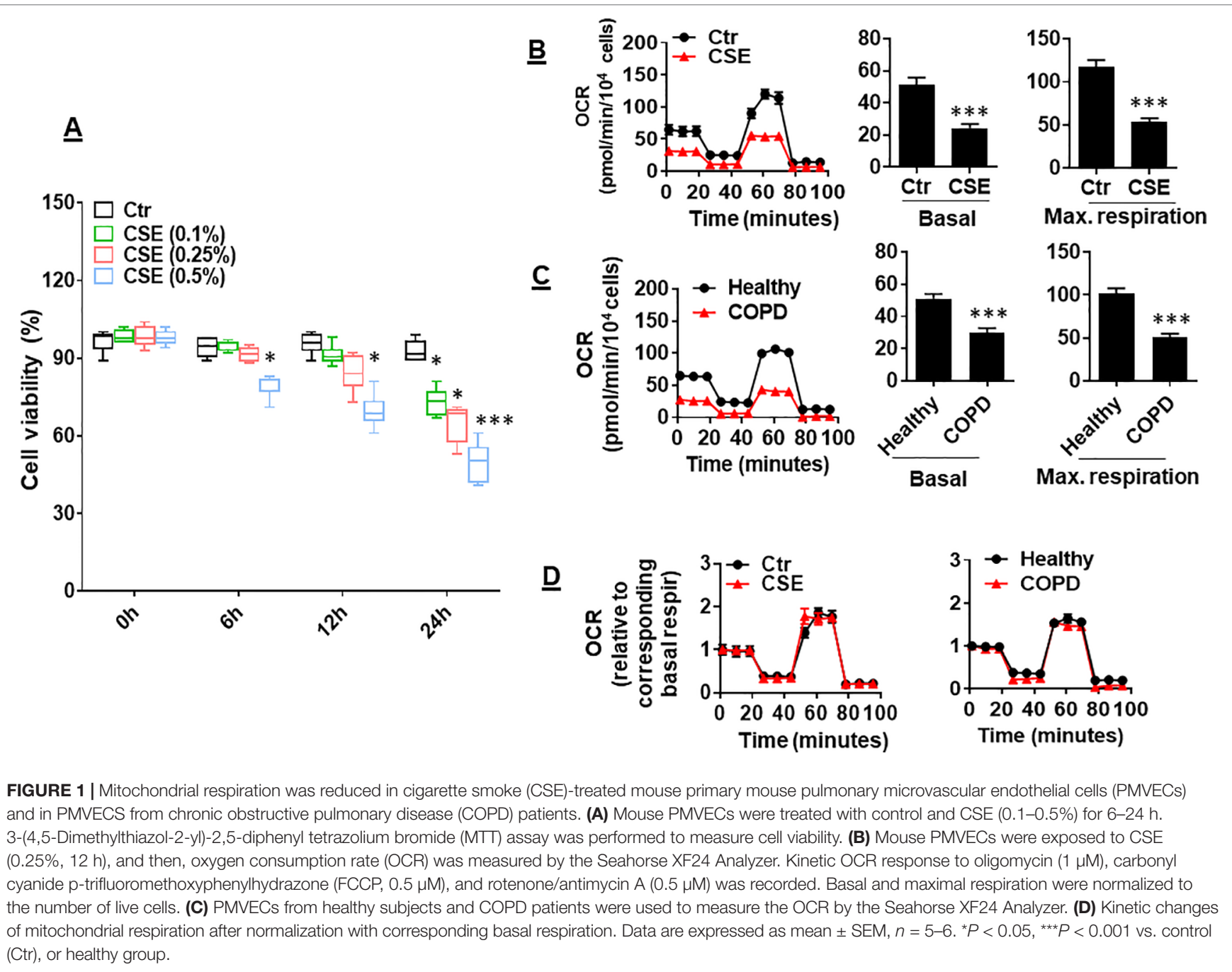



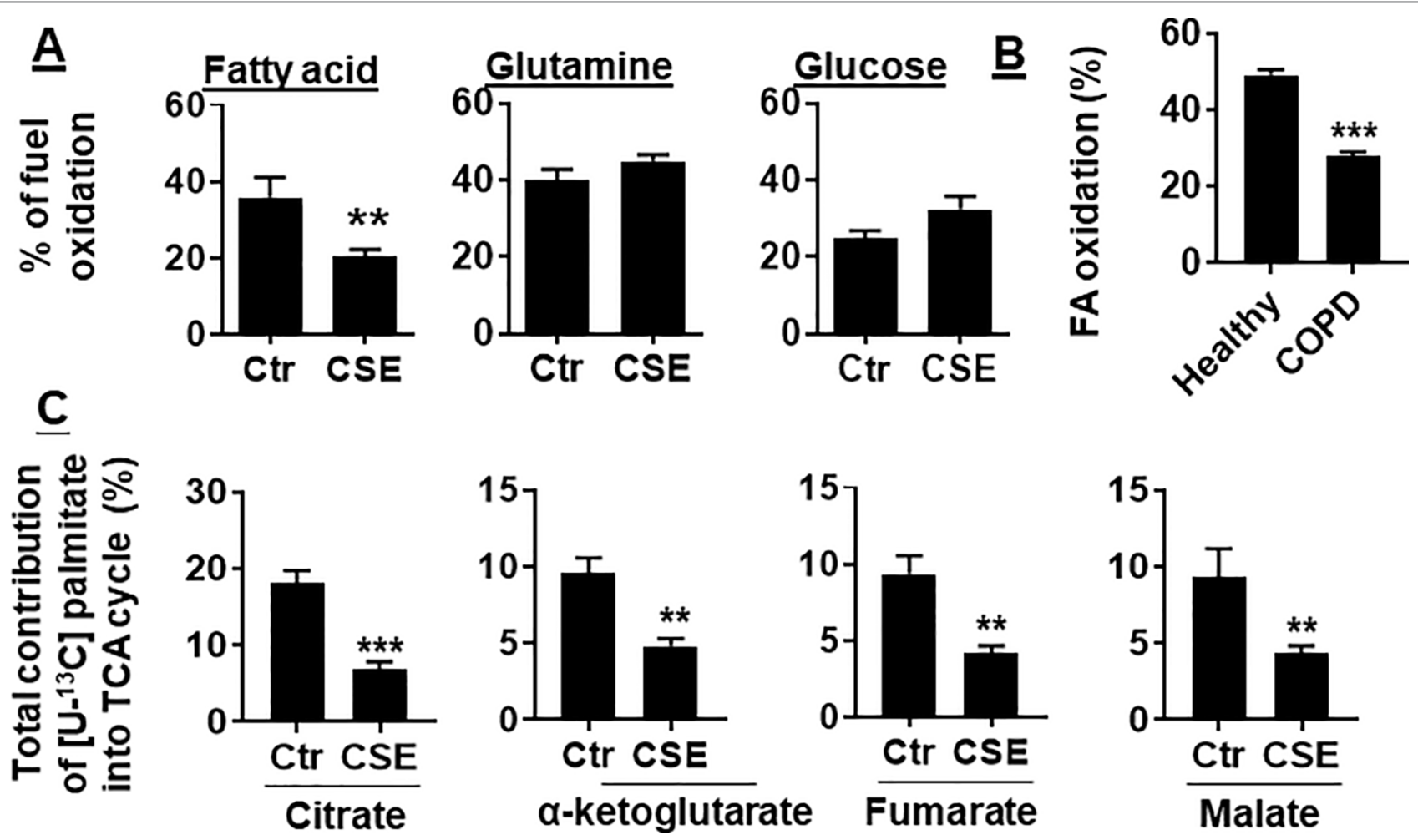

FIGURE 2 | FAO was reduced in CSE-treated mouse PMVECs and in PMVECs from COPD patients. (A) Mouse PMVECs were exposed to CSE (0.25\%) for $12 \mathrm{~h}$. Fuel utilization was measured in cells by the Seahorse XF24 Analyzer when the pathway inhibitors: etomoxir (4 $\mu \mathrm{M})$, BPTES ( $3 \mu \mathrm{M})$, and UK5099 ( $2 \mu \mathrm{M})$ were injected. The oxidation of FA, glutamine, and glucose was calculated as the difference of OCR between etomoxir, BPTES, or UK5099 and vehicle treatments, respectively. (B) FAO was measured in PMVECs from healthy subjects and COPD patients using the Seahorse XF24 Analyzer. (C) After treatment of mouse PMVECs with $(0.25 \%, 12 \mathrm{~h}), \mathrm{U}^{-13} \mathrm{C}$ palmitate labeled $(50 \mu \mathrm{M})$ was added for $24 \mathrm{~h}$. Levels of $13 \mathrm{C}$-labeled citrate, $\alpha$-ketoglutarate, fumarate, and malate were measured using mass spectrometry. Data are expressed as mean $\pm \mathrm{SEM}, n=5-6 .{ }^{\star \star} P<0.01,{ }^{\star \star \star} P<0.001$ vs. control (Ctr) or healthy group.

carnitine shuttle. Hence, we measured the mRNA levels of Cpt1 isoforms including Cpt 1a, Cpt 1b, and Cpt 1c. As shown in Figure 3A, Cptla mRNA was significantly reduced in mouse PMVECs treated with CSE. Similarly, CSE treatment decreased Cpt1a protein in mouse PMVECs (Figure 3B). The levels of Cpt1a mRNA and proteins were significantly reduced in PMVECs from patients with COPD when compared with healthy subjects (Figures 3C, D). These data suggest that CS-induced FAO reduction is due to the decreased levels of Cptla.

\section{FAO Augmentation Ameliorated CSE- Induced Apoptosis}

CS has been shown to cause apoptosis in lung ECs (Chen et al., 2012; Yang et al., 2015). It is not clear whether manipulation of FAO affects CS-induced apoptosis. To answer this question, we treated CSE $(0.25 \%, 12 \mathrm{~h})$-exposed mouse PMVECs with L-carnitine $(1 \mathrm{mM})$ or etomoxir $(10 \mu \mathrm{M})$ for $12 \mathrm{~h}$. As shown in Figure 4A, inhibition of Cpt1 by etomoxir reduced, whereas supplementing L-carnitine as a substrate of Cpt1 enhanced, the FAO in PMVECs in both control and CSE groups. The apoptosis determined by the Annexin V Apoptosis Detection kit was further increased in CSE-exposed PMVECs treated with etomoxir, and these effects were attenuated by L-carnitine (Figure 4B).
We next asked whether L-carnitine treatment alters Cptla expression. As expected, CSE treatment decreased Cptla gene expression (Figure 4C). L-Carnitine treatment increased expression of Cptla mRNA in both control and CSE groups (Figure 4C). We next silenced Cptla to determine whether this affects L-carnitine's effect on apoptosis. Transfection of Cptla siRNA significantly reduced Cptla gene expression (Figure 4D). Knockdown of Cptla increased Annexin V-positive cells, which was further augmented by CSE treatment (Figure 4E). Furthermore, Cptla silencing abolished L-carnitine's effect on apoptosis in response to CSE treatment (Figure 4F). All the results demonstrate that enhancing FAO attenuates CSE-induced apoptosis by upregulating Cptla in PMVECs.

\section{CSE Increased Ceramide Synthesis, Which Was Modulated by the FAO}

Ceramide is able to cause apoptosis in ECs (Paumen et al., 1997). We determined whether CSE increases ceramide synthesis, and this effect is modulated by the FAO. As shown in Figure 5A, CSE treatment increased the levels of C16:0 ceramide in PMVECs, which was reduced by L-carnitine $(1 \mathrm{mM})$. Next, we treated human PMVECs with $\mathrm{U}-{ }^{13} \mathrm{C}$-palmitate $(100 \mu \mathrm{M}, 6 \mathrm{~h})$ and determined palmitate-derived ceramide by mass spectrometry. 

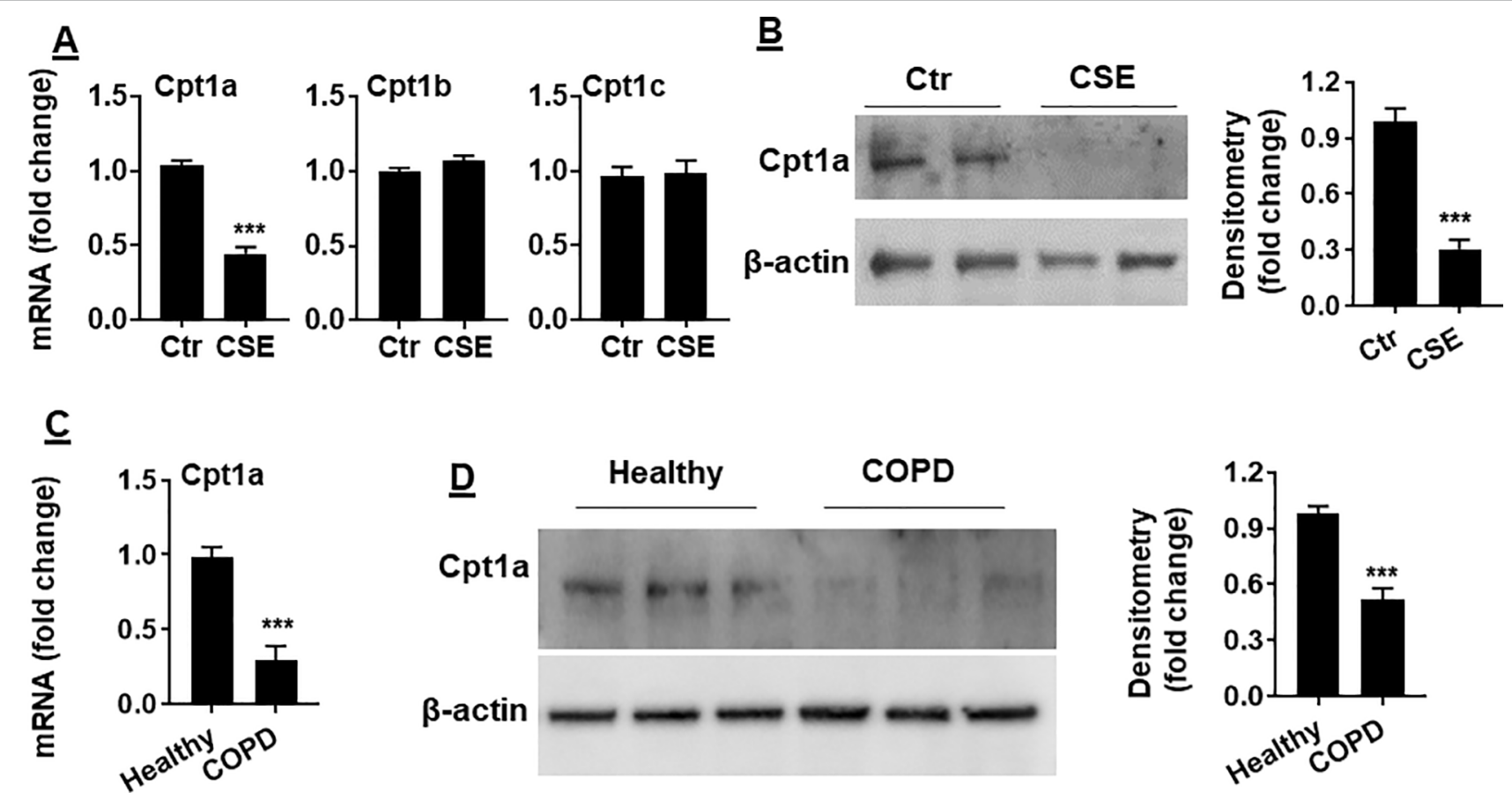

FIGURE 3 | Cpt1a levels were reduced in CSE-treated mouse PMVECs and in PMVECs from COPD patients. (A) Mouse PMVECs were treated with CSE (0.25\%, 12 h), RNA was extracted, and Cpt1a, Cpt1b, and Cpt1c mRNAs were measured by qRT-PCR. (B) Levels of Cpt1a protein were measured in mouse PMVECs treated with CSE by Western blot. (C) Cpt1a mRNA was measured by GRT-PCR in PMVECs from healthy subjects and COPD patients. (D) Levels of Cpt1a protein were measured in human PMVECs by Western blot. Data are expressed as mean $\pm \mathrm{SEM}, n=5-6 .{ }^{* \star *} P<0.001 \mathrm{vs}$. control (Ctr) or healthy group.

CSE treatment increased ${ }^{13} \mathrm{C}$-labeled ceramides, and these effects were attenuated when PMVECs were treated with L-carnitine (Figure 5B). In addition, we treated cells with a specific inhibitor of de novo ceramide synthesis (myriocin, $100 \mathrm{nM}$, $12 \mathrm{~h}$ ) and L-carnitine ( $1 \mathrm{mM}, 12 \mathrm{~h}$ ) in the presence of exogenous palmitate-BSA $(50 \mu \mathrm{M}, 12 \mathrm{~h})$. As shown in Figure 5C, treatment with exogenous palmitate significantly augmented apoptosis as detected by the Annexin V Apoptosis Detection kit compared with control. Treatments with L-carnitine exhibited a similar effect to myriocin in reducing palmitate-induced apoptosis in PMVECs (Figure 5C). Altogether, these results suggest that CS increases the conversion from fatty acids to ceramide, leading to apoptosis, and these effects are inhibited by enhancing FAO.

\section{Glycolysis Was Increased by CS Exposure, Which Was Not Associated With Apoptosis}

In ECs, glycolysis is the main metabolic pathway for generating bioenergetics. Thus, we determined whether CSE treatment alters glycolysis, and this associates with apoptosis. When mouse PMVECs were treated with CSE $(0.25 \%, 12 \mathrm{~h})$, glycolysis was increased as reflected by increased extracellular acidification rate (ECAR) (Figure 6A). Inhibition of glycolysis by 2-deoxy-D-glucose ( $3 \mathrm{mM}, 12 \mathrm{~h}$ ) had no effects on CSEinduced increase in Annexin V-positive cells (Figure 6B). The results suggest that CS-induced apoptosis in PMVECs is not associated with glycolysis.

\section{DISCUSSION}

Previous studies have proven that COPD patients have problems with their metabolism (Paige et al., 2011; Kao et al., 2012; Wang et al., 2013). There are no reports studying metabolic reprogramming in CS-induced apoptosis in lung EC. Here, we showed that CSE treatment reduced mitochondrial respiration in lung ECs, which were consistent with the results of decreased mitochondrial respiration in lung ECs by acrolein (Lu et al., 2017). Specifically, we also showed that CSE decreased FAO in lung ECs. This was associated with increased ceramide synthesis and apoptosis. Enhancing FAO by L-carnitine reduced, where inhibiting FAO by etomoxir or genetic knockdown of Cpt1a further augmented, CSE-induced apoptosis in lung ECs (Figure 7). Hence, CSE treatment reduces $\mathrm{FAO}$, leading to increased ceramide synthesis and apoptosis in lung ECs.

Compared to nonsmokers, primary basal stem/progenitor cells in the airways in healthy smoker had reduced metabolites in the tricarboxylic acid cycle, including acetyl-CoA, NADH, and FADH2 (Deeb et al., 2016). In contrast, an animal study has shown that CS exposure increased the levels of malate, citrate, and fumarate in rat lungs (Barupal et al., 2016). These studies suggest that CS may affect mitochondrial oxidative phosphorylation in a cell-specific manner. We found that CSE reduced mitochondrial respiration in lung ECs, which was determined by measuring the OCR via electron transport 

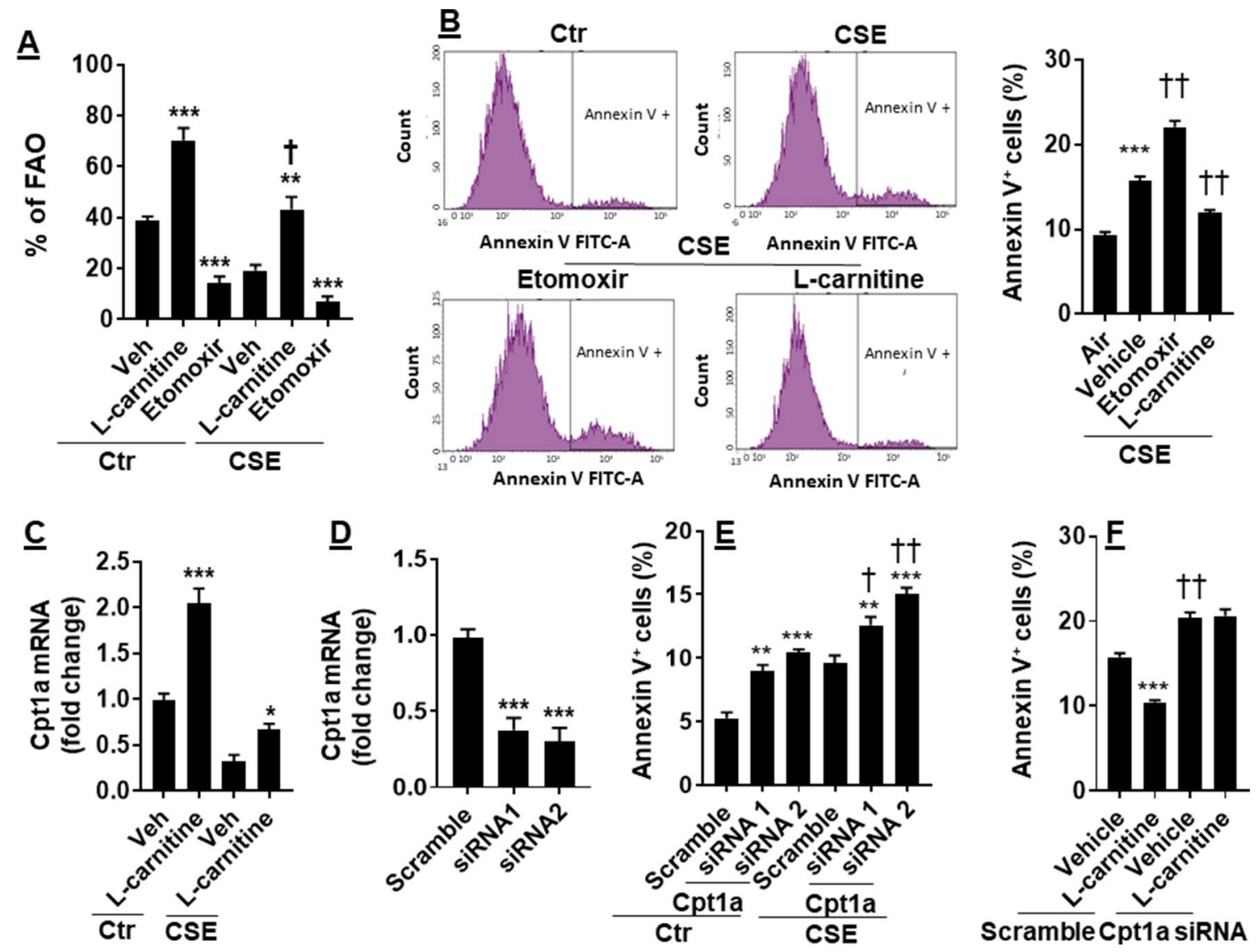

FIGURE 4 | Enhancing FAO attenuated CSE-induced apoptosis. Mouse PMVECs were treated with L-carnitine (1 mM, 12 h) or etomoxir (10 $\mu$ M, 12 h) in the absence or presence of CSE $\left(0.25 \%, 12\right.$ h). (A) FAO was measured by the Seahorse Analyzer. ${ }^{\star \star} P<0.01$, ${ }^{\star \star \star} P<0.001$ vs. vehicle (veh); ${ }^{\dagger} P<0.05$ vs. Ctr/L-carnitine. (B) Annexin $V^{+}$ cells were assessed by flow cytometry. ${ }^{* \star} P<0.001$ vs. air; ${ }^{\dagger} P<0.01$ vs. CSE/vehicle. (C) Cpt1a mRNA was measured by qRT-PCR. ${ }^{\star} P<0.05$, ${ }^{\star \star \star} P<0.001$ vs. vehicle (veh). (D) Mouse PMVECs were transfected with scramble or Cpt1a siRNA, and Cpt1a mRNA was measured by qRT-PCR. ${ }^{* \star *} P<0.001$ vs. scramble siRNA. (E) Annexin $\mathrm{V}^{+}$cells were assessed by flow cytometry in scramble or Cpt1a siRNA transfected cells in response to CSE $(0.25 \%, 12 \mathrm{~h})$ treatment. ${ }^{\star \star} P<0.01,{ }^{\star \star \star} P<0.001 \mathrm{vs}$. scramble siRNA; ${ }^{\dagger} P<0.05,{ }^{\dagger+} P<0.01$ vs. Ctr. (F) Cpt1a siRNA transfected cells were treated with $\mathrm{L}$-carnitine in response to CSE $(0.25 \%, 12 \mathrm{~h})$ treatment. Annexin $\mathrm{V}^{+}$cells were assessed by flow cytometry. ${ }^{\star \star \star} P<0.001$ vs. vehicle (veh); ${ }^{\dagger \dagger} P<0.01$ vs. scramble siRNA. Data are expressed as mean \pm SEM, $n=5-6$.

chain. A previous report has shown that CS blocked the mitochondrial respiratory chain in lung epithelial cells (Van Der Toorn et al., 2007). It is unknown whether CS impairs either the mitochondrial respiratory chain or tricarboxylic acid cycle, thereby reducing mitochondrial respiration in lung ECs. Further study is required to determine whether CS and it components impair oxidative phosphorylation in a dose- and time-dependent manner.

Cpt1 is a rate-limiting enzyme for carnitine shuttle during the FAO. There are a few studies showing that CS induced expression of Cpt1a, thereby enhancing FAO in lung epithelial cells and mouse lungs (Agarwal et al., 2014; Jiang et al., 2017). In contrast, FAO is impaired in alveolar epithelial cells during pathogen-induced acute lung injury (Cui et al., 2019). We found that CS exposure reduced Cpt1a levels and FAO in lung ECs. The discrepancies may be due to differences in epithelial cells, ECs, and lungs, as well as CS exposure durations. The limitation in our study is that we used commercial human PMVECs, which were not able to provide demographic information of subjects. Further study, using PMVECs from COPD patients with stratified demographic characteristics, including age, gender, smoking history, and disease severity, would reveal the effects of these features on Cptla and FAO during COPD progression.

We found that the levels of Cpt1b or Cpt1c were not detectable by Western blot in lung ECs (data not shown). This is in agreement with the report that Cptla is abundantly expressed, while Cpt1b or Cpt1c is expressed at very low levels in rat lungs (Ceccarelli et al., 2011). L-Carnitine is a metabolite critical for transporting long-chain fatty acids into the mitochondria for subsequent $\beta$-oxidation. It has been shown that $\mathrm{L}$-carnitine is reduced in mouse lungs with emphysema (Conlon et al., 2016). As a substrate of Cpt1, L-carnitine could increase Cpt1 gene expression, protein levels and activity (Karlic et al., 2002; Xi et al., 2008). This is in agreement with our finding that L-carnitine treatment increased Cptla gene expression in lung ECs. This contributes to the protection of L-carnitine against CSE-induced apoptosis. 

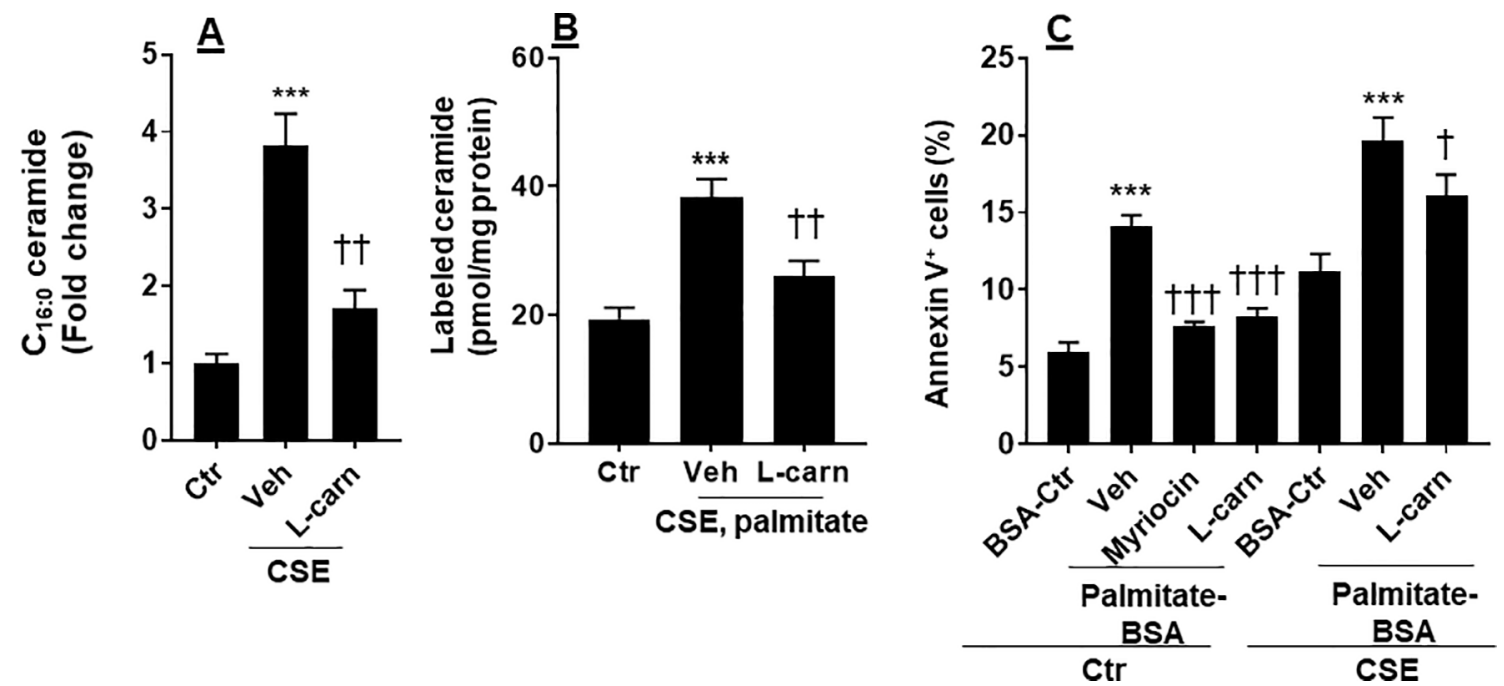

FIGURE 5 | CSE increased palmitate-derived ceramide leading to apoptosis. (A) Mouse PMVECs were treated with CSE (0.25\%, $12 \mathrm{~h})$ in the absence or presence of L-carnitine (1 mM, 12 h) treatment. Ceramide were measured in cells by mass spectrometry. (B) After CSE (0.25\%, $12 \mathrm{~h})$ along with L-carnitine (1 mM, $12 \mathrm{~h})$ treatment, $\mathrm{U}-{ }^{13} \mathrm{C}$ palmitate $(100 \mu \mathrm{M})$ was added for $6 \mathrm{~h}$ incubation. ${ }^{13} \mathrm{C}$-Ceramide was measured by mass spectrometry. (C) Mouse PMVECs were treated CSE (0.25\%, $\left.12 \mathrm{~h}\right)$ along with L-carnitine $(1 \mathrm{mM})$ and myriocin $(100 \mathrm{nM})$ for $12 \mathrm{~h}$ in the presence of palmitate-BSA or control BSA for $12 \mathrm{~h}$. Annexin V-positive cells were measured by flow cytometry. Data are expressed as mean $\pm \mathrm{SEM}, n=5-6$. ${ }^{\star \star \star} P<0.001$ vs. air control (Ctr); ${ }^{\dagger} P<0.05,{ }^{\dagger t} P<0.01,{ }^{t+t} P<0.001$ vs. CSE/vehicle or palmitate-BSA/vehicle (veh).
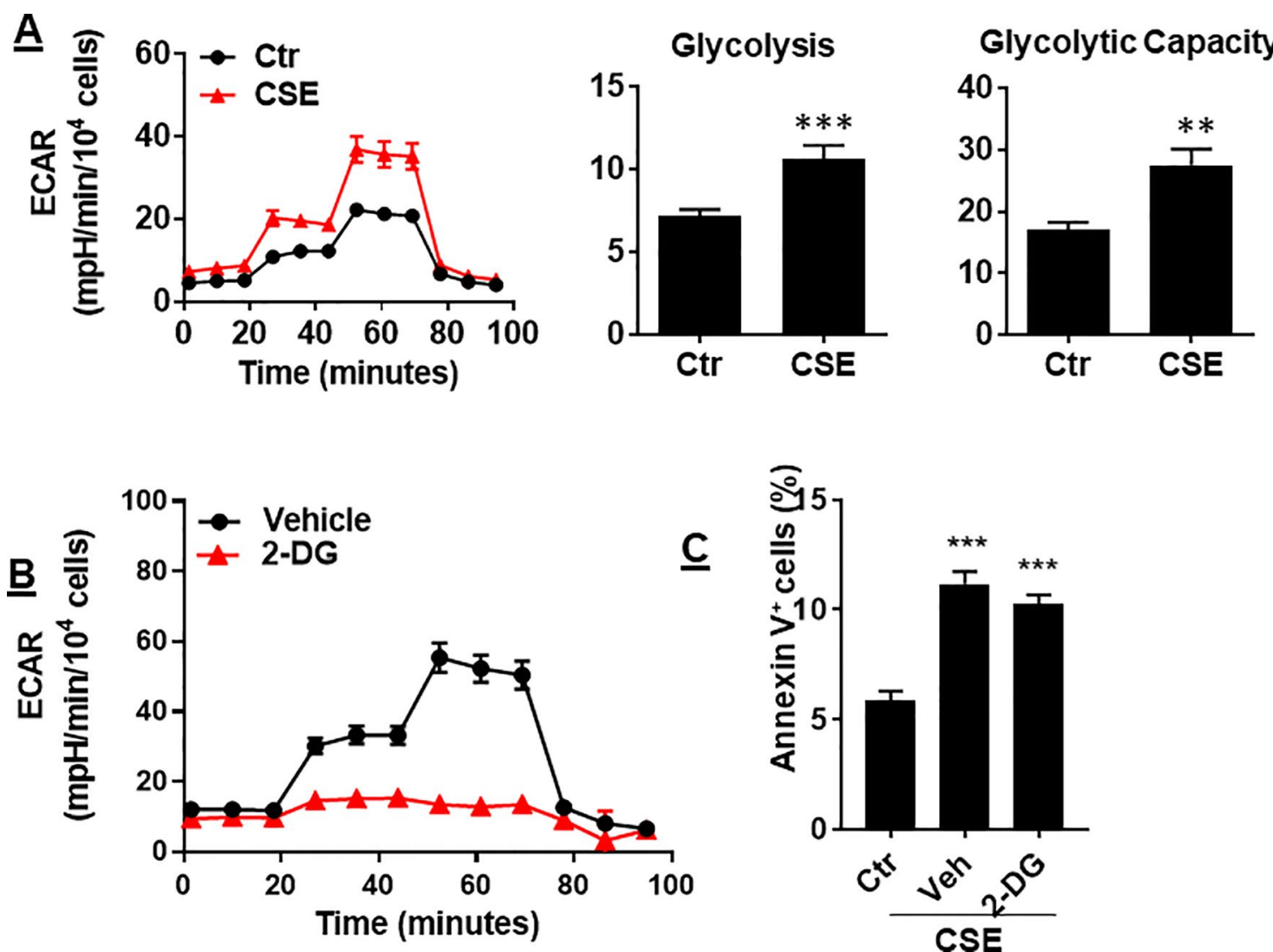

FIGURE 6 | CSE increased glycolysis, which was not associated with apoptosis. (A) Extracellular acidification rate (ECAR) was measured by the Seahorse Analyzer in mouse PMVECs exposed to CSE (0.25\%, 12 h). (B) Mouse PMVECs were treated with 2-DG (3 mM, 12 h), glycolysis was measured by the Seahorse Analyzer. (C) Mouse PMVECs were treated with 2-DG (3 mM, $12 \mathrm{~h})$ in the presence of CSE $(0.25 \%, 12 \mathrm{~h})$. Annexin $\mathrm{V}^{+}$cells were assessed by flow cytometry. Data are expressed as mean \pm SEM, $n=3-6$, ${ }^{\star \star} P<0.01,{ }^{\star \star \star} P<0.001$ vs. air control (Ctr). 


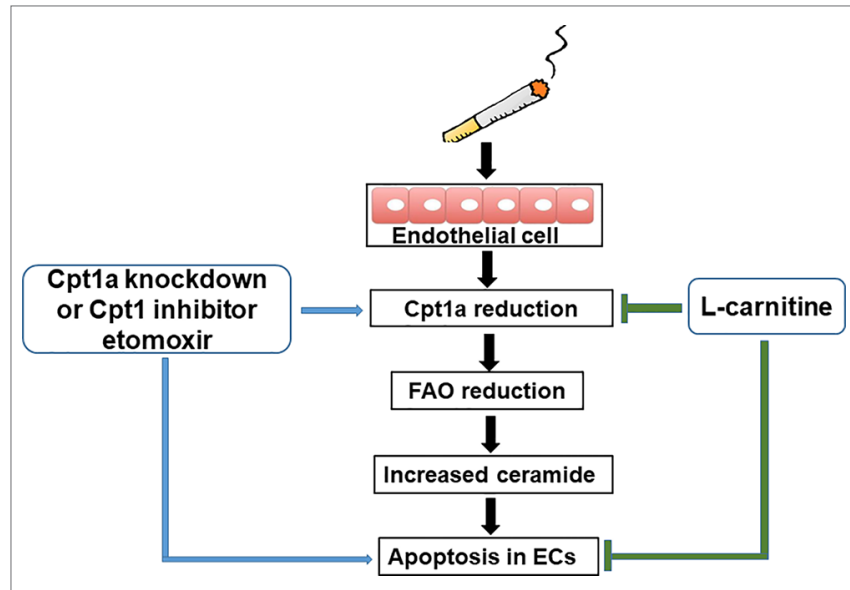

FIGURE 7 | A schematic figure summarizing the present study. Cigarette smoke causes apoptosis by downregulating fatty acid oxidation and increasing ceramide synthesis in lung endothelial cells.

The levels of sphingolipids, including ceramide, were significantly increased in sputum from smokers with COPD compared to smokers without COPD (Telenga et al., 2014). C16-Ceramide accumulation could cause mitochondrial damage and necroptosis in lung epithelial cells (Mizumura et al., 2018). This is in agreement with our findings that CS exposure increased levels of ceramide in lung ECs. Ceramide causes apoptosis and emphysema of lung ECs (Petrache et al., 2005; Garcia-Lucio et al., 2018). In fact, reducing ceramide synthesis by myriocin reduced CSE-induced apoptosis in lung ECs. The mechanisms underlying CS-induced increase in ceramide are associated with reduced FAO; however, it remains elusive whether Cptla knockdown affects CSE-induced increase in ceramide. This was evidenced by the findings that enhancing FAO by $\mathrm{L}$-carnitine reduced CS-induced ceramide synthesis and apoptosis in lung ECs. Whether FAO reduction also mediates necroptosis in lung ECs exposed to CS remains elusive. L-Carnitine administration attenuates elastase-induced pulmonary emphysema (Conlon et al., 2016). A clinical study has shown that carnitine can improve exercise tolerance and inspiratory muscle strength in COPD patients (Borghi-Silva et al., 2006). Altogether, these findings suggest that enhancing FAO by L-carnitine may be beneficial to COPD/emphysema.

A previous report has shown that glycolysis was reduced in in the CS-exposed alveolar type II cells (Agarwal et al.,

\section{REFERENCES}

Agarwal, A. R., Yin, F., and Cadenas, E. (2014). Short-term cigarette smoke exposure leads to metabolic alterations in lung alveolar cells. Am. J. Respir. Cell. Mol. Biol. 51, 284-293. doi: 10.1165/rcmb.2013-0523OC

Barupal, D. K., Pinkerton, K. E., Hood, C., Kind, T., and Fiehn, O. (2016). Environmental tobacco smoke alters metabolic systems in adult rats. Chem. Res. Toxicol. 29, 1818-1827. doi: 10.1021/acs.chemrestox.6b00187

Borghi-Silva, A., Baldissera, V., Sampaio, L. M., Pires-Dilorenzo, V. A., Jamami, M., Demonte, A., et al. (2006). L-Carnitine as an ergogenic aid for patients
2014). We found that CSE treatment increased glycolysis in lung ECs. It remains elusive whether these cells may attempt to restore glycolysis to generate ATP or lactate to feed the tricarboxylic acid cycle as a compensatory mechanism against reduced mitochondrial respiration for bioenergetics and cellular function (Hui et al., 2017). Inhibition of glycolysis with 2-deoxy-D-glucose had no significant effect on CSEinduced apoptosis. Hence, glycolysis is not involved in CSEinduced apoptosis in lung ECs.

In conclusion, CSE treatment reduced mitochondrial respiration and FAO, leading to apoptosis in lung ECs. The mechanisms underlying CSE-induced apoptosis is associated with increased ceramide synthesis in condition of insufficient FAO. L-Carnitine enhances FAO but attenuates CSE-induced ceramide levels and apoptosis by upregulating Cptla in lung ECs. Therefore, the reduction in FAO in lung ECs may contribute to pathogenesis of CS-induced COPD/emphysema.

\section{DATA AVAILABILITY}

The raw data supporting the conclusions of this manuscript will be made available by the authors, without undue reservation, to any qualified researcher.

\section{ETHICS STATEMENT}

The animal study was approved by the Animal Care and Ethics Committee of the second Hospital of Shanxi Medical School (CMTT\#: 2013012) in accordance with international standard.

\section{AUTHOR CONTRIBUTIONS}

Conception and design: HWY and JL; data acquisition and analysis: JG, HZ, TL, LL, EC, SZ, and LK; data interpretation: JG, HWY, and JL; drafting the manuscript: JG; revising the manuscript: JG, HWY, and JL.

\section{FUNDING}

This work was supported by the Education Department of Shanxi Province Foundation for Graduate Education Innovation (\#2018BY068) and Health Commission of Shanxi Province Foundation for Higher Education Technology Innovation (\#2017050). 
Chen, Y., Luo, H., Kang, N., Guan, C., Long, Y., Cao, J., et al. (2012). Beraprost sodium attenuates cigarette smoke extract-induced apoptosis in vascular endothelial cells. Mol. Biol. Rep. 39, 10447-10457. doi: 10.1007/s11033-012-1924-1

Conlon, T. M., Bartel, J., Ballweg, K., Gunter, S., Prehn, C., Krumsiek, J., et al. (2016). Metabolomics screening identifies reduced L-carnitine to be associated with progressive emphysema. Clin. Sci. (Lond.) 130, 273-287. doi: 10.1042/CS20150438

Cruickshank-Quinn, C. I., Jacobson, S., Hughes, G., Powell, R. L., Petrache, I., Kechris, K., et al. (2018). Metabolomics and transcriptomics pathway approach reveals outcome-specific perturbations in COPD. Sci. Rep. 8, 17132. doi: 10.1038/s41598-018-35372-w

Cui, H., Xie, N., Banerjee, S., Ge, J., Guo, S., and Liu, G. (2019). Impairment of fatty acid oxidation in alveolar epithelial cells mediates acute lung injury. Am. J. Respir. Cell. Mol. Biol. 60, 167-178. doi: 10.1165/rcmb.2018-0152OC

Cui, W., Zhang, Z., Zhang, P., Qu, J., Zheng, C., Mo, X., et al. (2018). Nrf2 attenuates inflammatory response in COPD/emphysema: crosstalk with Wnt3a/betacatenin and AMPK pathways. J. Cell. Mol. Med. 22, 3514-3525. doi: 10.1111/ jcmm. 13628

Deeb, R. S., Walters, M. S., Strulovici-Barel, Y., Chen, Q., Gross, S. S., and Crystal, R. G. (2016). Smoking-associated disordering of the airway basal stem/progenitor cell metabotype. Am. J. Respir. Cell. Mol. Biol. 54, 231-240. doi: 10.1165/rcmb.2015-0055OC

Garcia-Lucio, J., Peinado, V. I., De Jover, L., Del Pozo, R., Blanco, I., Bonjoch, C., et al. (2018). Imbalance between endothelial damage and repair capacity in chronic obstructive pulmonary disease. PLoS One 13, e0195724. doi: 10.1371/ journal.pone.0195724

Giordano, R. J., Lahdenranta, J., Zhen, L., Chukwueke, U., Petrache, I., Langley, R. R., et al. (2008). Targeted induction of lung endothelial cell apoptosis causes emphysema-like changes in the mouse. J. Biol. Chem. 283, 29447-29460. doi: 10.1074/jbc.M804595200

Hui, S., Ghergurovich, J. M., Morscher, R. J., Jang, C., Teng, X., Lu, W., et al. (2017). Glucose feeds the TCA cycle via circulating lactate. Nature 551, 115-118. doi: 10.1038 /nature24057

Ivanisevic, J., Zhu, Z. J., Plate, L., Tautenhahn, R., Chen, S., O'brien, P. J., et al. (2013). Toward 'omic scale metabolite profiling: a dual separation-mass spectrometry approach for coverage of lipid and central carbon metabolism. Anal. Chem. 85, 6876-6884. doi: 10.1021/ac401140h

Jiang, Z., Knudsen, N. H., Wang, G., Qiu, W., Naing, Z. Z. C., Bai, Y., et al. (2017). Genetic control of fatty acid beta-oxidation in chronic obstructive pulmonary disease. Am. J. Respir. Cell. Mol. Biol. 56, 738-748. doi: 10.1165/ rcmb.2016-0282OC

Kao, C. C., Hsu, J. W., Bandi, V., Hanania, N. A., Kheradmand, F., and Jahoor, F. (2012). Glucose and pyruvate metabolism in severe chronic obstructive pulmonary disease. J. Appl. Physiol. (1985) 112, 42-47. doi: 10.1152/ japplphysiol.00599.2011

Karlic, H., Lohninger, S., Koeck, T., and Lohninger, A. (2002). Dietary l-carnitine stimulates carnitine acyltransferases in the liver of aged rats. J. Histochem. Cytochem. 50, 205-212. doi: 10.1177/002215540205000208

Kim, S. Y., Kim, H. J., Park, M. K., Huh, J. W., Park, H. Y., Ha, S. Y., et al. (2016). Mitochondrial E3 ubiquitin protein ligase 1 mediates cigarette smoke-induced endothelial cell death and dysfunction. Am. J. Respir. Cell. Mol. Biol. 54, 284296. doi: 10.1165/rcmb.2014-0377OC

Lu, Q., Mundy, M., Chambers, E., Lange, T., Newton, J., Borgas, D., et al. (2017). Alda-1 protects against acrolein-induced acute lung injury and endothelial barrier dysfunction. Am. J. Respir. Cell. Mol. Biol. 57, 662-673. doi: 10.1165/ rcmb.2016-0342OC

Mirza, S., Clay, R. D., Koslow, M. A., and Scanlon, P. D. (2018). COPD guidelines: a review of the 2018 GOLD Report. Mayo Clin. Proc. 93, 1488-1502. doi: 10.1016/j.mayocp.2018.05.026

Mizumura, K., Justice, M. J., Schweitzer, K. S., Krishnan, S., Bronova, I., Berdyshev, E. V., et al. (2018). Sphingolipid regulation of lung epithelial cell mitophagy and necroptosis during cigarette smoke exposure. FASEB J. 32, 1880-1890. doi: 10.1096/f.201700571R

Paige, M., Burdick, M. D., Kim, S., Xu, J., Lee, J. K., and Shim, Y. M. (2011). Pilot analysis of the plasma metabolite profiles associated with emphysematous chronic obstructive pulmonary disease phenotype. Biochem. Biophys. Res. Commun. 413, 588-593. doi: 10.1016/j.bbrc.2011.09.006

Paumen, M. B., Ishida, Y., Muramatsu, M., Yamamoto, M., and Honjo, T. (1997). Inhibition of carnitine palmitoyltransferase I augments sphingolipid synthesis and palmitate-induced apoptosis. J. Biol. Chem. 272, 3324-3329. doi: 10.1074/ jbc. 272.6 .3324

Petrache, I., Natarajan, V., Zhen, L., Medler, T. R., Richter, A. T., Cho, C., et al. (2005). Ceramide upregulation causes pulmonary cell apoptosis and emphysema-like disease in mice. Nat. Med. 11, 491-498. doi: 10.1038/nm1238

Pike Winer, L. S., and Wu, M. (2014). Rapid analysis of glycolytic and oxidative substrate flux of cancer cells in a microplate. PLoS One 9, e109916. doi: 10.1371/ journal.pone.0109916

Rennard, S. I., and Drummond, M. B. (2015). Early chronic obstructive pulmonary disease: definition, assessment, and prevention. Lancet 385, 1778-1788. doi: 10.1016/S0140-6736(15)60647-X

Suresh, K., Servinsky, L., Reyes, J., Undem, C., Zaldumbide, J., Rentsendorj, O., et al. (2017). CD36 mediates H2O2-induced calcium influx in lung microvascular endothelial cells. Am. J. Physiol. Lung Cell. Mol. Physiol. 312, L143-L153. doi: 10.1152/ajplung.00361.2016

Telenga, E. D., Hoffmann, R. F., Ruben, T. K., Hoonhorst, S. J., Willemse, B. W., Van Oosterhout, A. J., et al. (2014). Untargeted lipidomic analysis in chronic obstructive pulmonary disease. Uncovering sphingolipids. Am. J. Respir. Crit. Care Med. 190, 155-164. doi: 10.1164/rccm.201312-2210OC

Van Der Toorn, M., Slebos, D. J., De Bruin, H. G., Leuvenink, H. G., Bakker, S. J., Gans, R. O., et al. (2007). Cigarette smoke-induced blockade of the mitochondrial respiratory chain switches lung epithelial cell apoptosis into necrosis. Am. J. Physiol. Lung Cell. Mol. Physiol. 292, L1211-L1218. doi: 10.1152/ ajplung.00291.2006

Vaskovsky, V. E., Kostetsky, E. Y., and Vasendin, I. M. (1975). A universal reagent for phospholipid analysis. J. Chromatogr. 114, 129-141. doi: 10.1016/ S0021-9673(00)85249-8

Voelkel, N. F. (2018). Cigarette smoke is an endothelial cell toxin. Am. J. Respir. Crit. Care Med. 197, 274. doi: 10.1164/rccm.201706-1123LE

Wang, L., Tang, Y., Liu, S., Mao, S., Ling, Y., Liu, D., et al. (2013). Metabonomic profiling of serum and urine by (1)H NMR-based spectroscopy discriminates patients with chronic obstructive pulmonary disease and healthy individuals. PLoS One 8, e65675. doi: 10.1371/journal.pone.0065675

Xi, L., Brown, K., Woodworth, J., Shim, K., Johnson, B., and Odle, J. (2008). Maternal dietary L-carnitine supplementation influences fetal carnitine status and stimulates carnitine palmitoyltransferase and pyruvate dehydrogenase complex activities in swine. J. Nutr. 138, 2356-2362. doi: 10.3945/jn.108.095638

Yang, M., Chen, P., Peng, H., Zhang, H., Chen, Y., Cai, S., et al. (2015). Cigarette smoke extract induces aberrant cytochrome-c oxidase subunit II methylation and apoptosis in human umbilical vascular endothelial cells. Am. J. Physiol. Cell. Physiol. 308, C378-C384. doi: 10.1152/ajpcell.00197.2014

Yao, C. H., Fowle-Grider, R., Mahieu, N. G., Liu, G. Y., Chen, Y. J., Wang, R., et al. (2016). Exogenous fatty acids are the preferred source of membrane lipids in proliferating fibroblasts. Cell. Chem. Biol. 23, 483-493. doi: 10.1016/j. chembiol.2016.03.007

Yao, H., Gong, J., Peterson, A. L., Lu, X., Zhang, P., and Dennery, P. A. (2019). Fatty acid oxidation protects against hyperoxia-induced endothelial cell apoptosis and lung injury in neonatal mice. Am. J. Respir. Cell. Mol. Biol. 60, 667-677. doi: 10.1165/rcmb.2018-0335OC

Yao, H., and Rahman, I. (2011). Current concepts on oxidative/carbonyl stress, inflammation and epigenetics in pathogenesis of chronic obstructive pulmonary disease. Toxicol. Appl. Pharmacol. 254, 72-85. doi: 10.1016/j. taap.2009.10.022

Yue, L., and Yao, H. (2016). Mitochondrial dysfunction in inflammatory responses and cellular senescence: pathogenesis and pharmacological targets for chronic lung diseases. Br. J. Pharmacol. 173, 2305-2318. doi: 10.1111/bph.13518

Conflict of Interest Statement: The authors declare that the research was conducted in the absence of any commercial or financial relationships that could be construed as a potential conflict of interest.

Copyright (c) 2019 Gong, Zhao, Liu, Li, Cheng, Zhi, Kong, Yao and Li. This is an open-access article distributed under the terms of the Creative Commons Attribution License (CC BY). The use, distribution or reproduction in other forums is permitted, provided the original author(s) and the copyright owner(s) are credited and that the original publication in this journal is cited, in accordance with accepted academic practice. No use, distribution or reproduction is permitted which does not comply with these terms. 